\title{
Study of Automatic PCB Jack Center Positioning System Based on Machine Vision
}

\author{
Jun-tao Xiong ${ }^{1}$, Jin-zhao $\mathrm{Li}^{1}$, Bao-xia $\mathrm{Sun}^{2}$, Jian-guo $\mathrm{Chen}^{{ }^{*}}$ \\ 1.College of Informatics, South China Agricultural University, Guangzhou 510642, China; \\ 2.Guangdong engineering polytechnic \\ *corresponding author, e-mail: 309283022@qq.com
}

\begin{abstract}
Make a study of real-time, automatic positioning of Printed Circuit Board (PCB) jack centers. Bwlabel algorithm is used to deal with the PCB images under the condition of backlighting. According to the feature of Bwlabel algorithm extract and match the jack center indexes for every component, calculate the jack center connection angle, and generate jack center positioning data, figuring out the jack center coordinates. Combining with camera parameters, both the radial and tangential lens distortion are corrected, revising the coordinates of jack centers. At last, an automatic PCB jack positioning system is developed.The system experiment results show that the requirement of production has been met with accuracy of positioning that minimum jack diameter of $2.32 \mathrm{~mm}$ and average time of $0.21 \mathrm{~s}$, which provides coordinates information for automatic assembly operation by manipulator.
\end{abstract}

Keywords: PCB, Bwlabel, distortion correction, jack center indexes, automatic positioning.

\section{Introduction}

Electronics manufacturing enterprises are typically Labor-intensive. Nowadays these enterprises are confronted with multiple pressures, such as high staff mobility, high labor costs, and low product added value (National Development and Reform Commission, 2008). In PCB assembly process, only few electronic components can be assembled by semiautomatic method with plug-in machine, most of them are assembled by the workers artificially. To change this situation, automation transformation is necessary for electronics manufacturing enterprises.

Now machine vision technique is widely used in electronics manufacturing with its own advantages - rapid, intelligent, precious, which plays an important role no matter in accurate positioning of the positioning jacks when making a PCB (He Wei-zhong, 2006; Chen Ke-yong, 2007), in error correction of the PCB relative position in SMT (Xia Qi, Zhou Ming-cai, WANG Hong-sheng, et al, 2004; Wang Hong, Wang Shi-gang, Xu Wei, et al, 2001; Jiang Tao, Wang An-lin, Wang Shi-gang, et al, 2005), or in PCB quality detection (Huang Yong-lin, 2010; Zhu Yan, Duan Zhe-min, 2007; Xie Hong-fei, Zhang Xian-ming, Kuang Yong-cong, et al, 2011; Su Lei, Zhang Guang-ming, Wei Xiao-dong, 2009).

Currently most enterprises obtain the PCB jacks coordinates data from the Protel software. However, it is tedious work to enter the jack coordinates one by one and manage the PCB files. To get rid of the PCB files, take images of PCB that is under the condition of backlighting with a CMOS camera. Extract and calculate the coordinates of jack centers, correcting the lens distortion, match and save the jack indexes, which can be reuse in mass 
production. The research not only can achieve the real-time, automatic positioning of PCB jack centers, but also lay the foundation for automatic assembly operation by manipulator.

\section{System Component}

Monocular vision system (see Fig. 1) is used to extract the coordinates of PCB jacks. The system is consisted of a DH-HV1310FC camera with resolution $1280 * 1024$, which connects the computer through IEEE1394 interface, a Computar M1614 optical lens with focal length $16 \mathrm{~mm}$, an OPT- 150150 white bottom-emitting light source and a computer with Windows XP OS, which runs an automatic positioning system based on Matlab R2009a platform.

Moreover, the calibration experiment should be done before using the camera and lens to take images. Use the Matlab calibration toolbox and chessboard template to do the calibration experiment (Jean-Yves Bouguet, 2010), figuring out the camera inner parameters (see Table. 1). Do not need to measure the external parameters because there is only one camera in use.

The calibration result shows that the lens has distortion, which may cause abnormal deformation when objects image in the photosensitive chip, reducing the compute accuracy, so in practice, the image processing result should be corrected.

Table 1. Camera calibration results [pixel]

\begin{tabular}{cc}
\hline $\begin{array}{c}\text { Parameter } \\
\text { name }\end{array}$ & Calibration value \\
\hline $\begin{array}{c}\text { Focal length } \\
\text { (fc) }\end{array}$ & {$[3120.30506,3132.0529]$} \\
$\begin{array}{c}\text { Principle point } \\
\text { (cc) }\end{array}$ & {$[482.91000,563.56912]$} \\
Skew & 0.00000 \\
(alpha_c) & {$[-$} \\
Distortion (kc) & $0.24935,1.35948,0.00553$, \\
& $-0.007990 .00000]$ \\
\hline
\end{tabular}

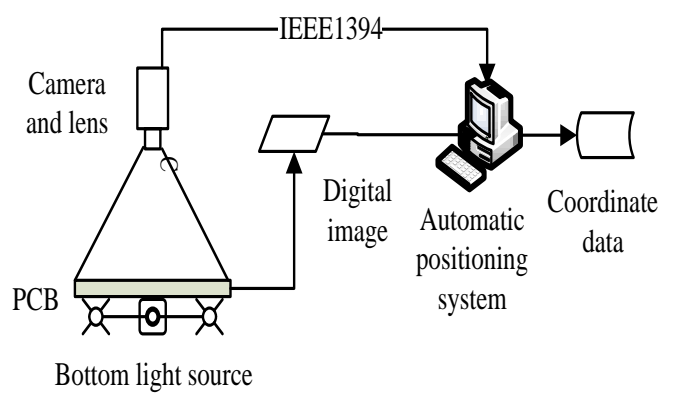

Figure 1. Schematic of system constitution

\section{PCB Jack Center Positioning algorithm based on Machine Vision}

\subsection{Bwlabel algorithm}

Bwlabel algorithm is used to identify and count the number of the white area in a binary image (Fishgumuxing, 2010) (see Fig. 2).

Suppose there is a binary image (see Fig. 2a). Firstly scan the image from left to right, top to bottom, count the number of "pixel groups", which cluster together with the gray value 1 , and calculate the sum. Secondly set the column number $\mathrm{c}[\mathrm{k}]$, start row number $\mathrm{sr}[\mathrm{k}]$ and end row number er[k] where a "pixel group" lays. Then scan the image again and label every "pixel group" begin with 1 . If there are 2 connected "pixel groups" in 2 adjacent columns, in fact they belong to a same one, which should be labeled the same index (see Fig. 2b). After that, the centroid of every white area can be calculated, which is equivalent to central coordinates. 


\subsection{Automatic positioning method}

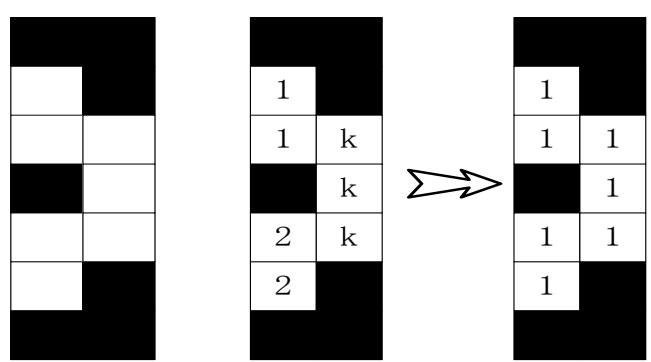

Fig. 2 Schematic of Bwlabel algorithm

\subsubsection{Obtain PCB images}

Fig. 3a shows the GM127Z003-G type PCB image under the condition of backlighting and $300 \mathrm{~mm}$ working distance. The white area is the PCB jacks whereas other area becomes gloomy because the light cannot pass through. Use Ostu algorithm (Wang Li, Zhang Maoqing, Wang Hong-dong, 2009; Rafael C. Gonzalez, Richard E. Woods, Steven L. Eddins, 2009) can easily binarize the image (see Fig. 3b), which highlights the PCB jacks.

\subsubsection{Identify jack centers with Bwlabel}

As for the binary image like what Fig. 3b shows, the central coordinates of every white area should be worked out. The shape of every white area is not regular, and usual circle detection algorithm cannot fit very well (Li Ting, Liu Ning, 2012), so the Bwlabel algorithm is used directly. The result saves the central coordinates and corresponding index of every white area (see Fig. 4).

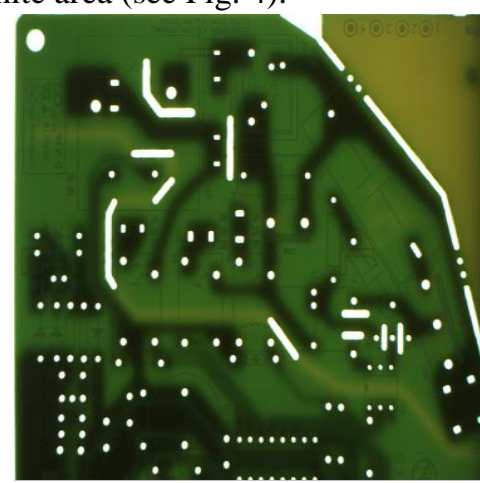

a

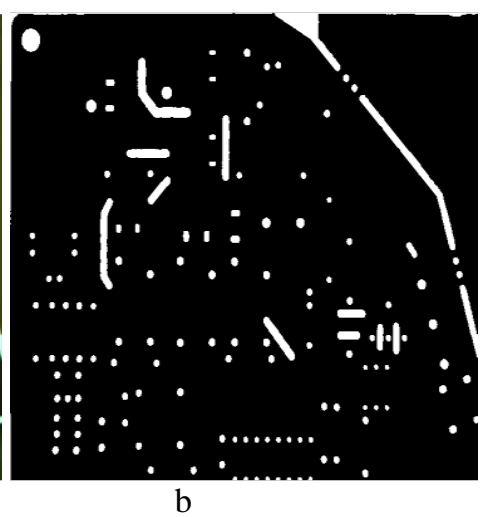

Fig. 3 PCB images

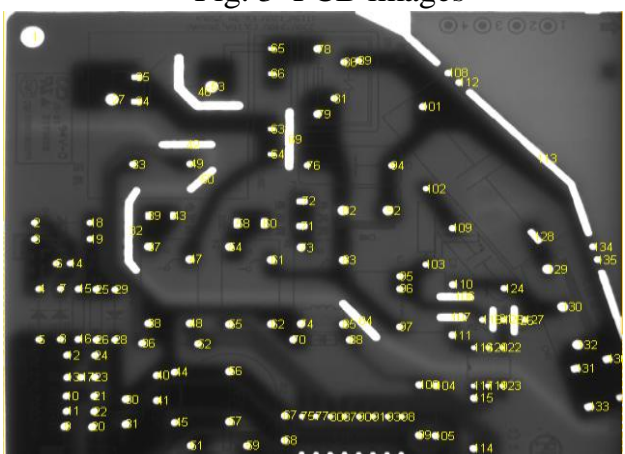

Fig. 4 Algorithm result 


\subsection{Distortion correction and error analysis}

As we know from Table. 1, the lens distortion can cause image deformation, which reduces the accuracy of jack coordinates. In this section, the radial and tangential distortion (Gary Bradski, Adrian Kaehler, 2009; Liu Rui-zhen, Yu Shi-qi, 2007) is referred to correct the coordinates of every jack center.

Suppose the jack center coordinates calculated by Bwlabel are (xd, yd), the ones after correction by the distortion model are (xn, yn). Refer to the parameters in Table. 1, equations are shown as follows:

$$
\begin{aligned}
& {\left[\begin{array}{l}
x_{n} \\
y_{n}
\end{array}\right]=\left(1+k c(1) r^{2}+k c(2) r^{4}+k c(5) r^{6}\right)\left[\begin{array}{l}
x_{d} \\
y_{d}
\end{array}\right]+d x} \\
& d x=\left[\begin{array}{l}
2 k c(3) x y+k c(4)\left(r^{2}+2 x^{2}\right) \\
k c(3)\left(r^{2}+2 y^{2}\right)+2 k c(4) x y
\end{array}\right] \\
& x=\frac{x_{d}-\operatorname{cc}(1)}{f c(1)}, y=\frac{y_{d}-c c(2)}{f c(2)}, r=\sqrt{x^{2}+y^{2}}
\end{aligned}
$$

Pay attention that the results derived from the formulas above are distance on the image (D.Im), whose unit is pixel. They can be transformed to actual distance (D.Ac), whose unit is $\mathrm{mm}$, according to the size of camera field of view (FOV) and resolution (R). The equation is shown below:

$$
D . A c=D . \operatorname{Im} \times F O V / R
$$

Calculate the difference between (xn, yn) and the actual coordinates of jack centers (X, Y) in both $\mathrm{x}$ and $\mathrm{y}$ direction, then compute the actual deviation $\Delta d$, equation is shown below and the results are shown as Table. 2 . Only 5 jacks are listed because of space limitations.

$$
\Delta \mathrm{d}=\sqrt{\left(\mathrm{x}_{\mathrm{n}}-\mathrm{X}\right)^{2}+\left(\mathrm{y}_{\mathrm{n}}-\mathrm{Y}\right)^{2}}
$$

As for the data in $\Delta d$ column in Table. 2 , the mean $\overline{\Delta d}$ is $0.48 \mathrm{~mm}$, standard deviation s is $0.28 \mathrm{~mm}$. Fig. 5 shows how to describe the accuracy of the results. The pin-diameter of electronic component is d, approximately $0.5-0.8 \mathrm{~mm} .2(\overline{\Delta d}+s)$ means the max difference between $(\mathrm{xn}, \mathrm{yn})$ and $(\mathrm{X}, \mathrm{Y})$.

Table. 2 Coordinate error with working distance of $300 \mathrm{~mm}$

\begin{tabular}{cccccc}
\hline $\begin{array}{c}\text { Jack } \\
\text { indexes }\end{array}$ & \multicolumn{2}{c}{ Coordinate } & \multicolumn{2}{c}{ Corrected coordinates } & Deviation \\
& $\mathrm{X}$ & $\mathrm{Y}$ & $\mathrm{Xc}$ & $\mathrm{Yc}$ & $\Delta \mathrm{d}$ \\
\hline 1 & 0 & 0 & 0 & 0 & 0 \\
2 & 14.4 & 12.4 & 14.32 & 12.52 & 0.15 \\
3 & 21.4 & 41.5 & 20.92 & 42.09 & 0.76 \\
4 & 32.5 & 10.5 & 32.23 & 10.01 & 0.56 \\
5 & 35.8 & 66.4 & 35.47 & 67.05 & 0.73 \\
$\ldots$ & $\ldots$ & $\ldots$ & $\ldots$ & $\ldots$ & $\ldots$ \\
\hline
\end{tabular}

D represents the actual radium of a jack on PCB. The compute accuracy has met the requirement of actual $\mathrm{PCB}$ assembly production if the statement shown below is true.

$$
\mathrm{D} \geq 2(\overline{\Delta \mathrm{d}}+\mathrm{s})+\mathrm{d}
$$

Substitute the results mentioned above into Eq. 6 and get $D \geq 2.32 \mathrm{~mm}$, which shows that the diameter of a jack have to more than $2.32 \mathrm{~mm}$, otherwise, the pins of electronic 
component possibly cannot insert into the jack. In practical production, pins diameter of electronic component is $2-3 \mathrm{~mm}$, so the accuracy has met the requirement basically.

Besides, as for real-time, apply the Bwlabel algorithm to the image Fig. 3a, which has 136 jacks totally, and calculate the corrected central coordinates of every jack. Run the process 20 times, with average time $0.21 \mathrm{~s}$. The real-time requirement has been met.

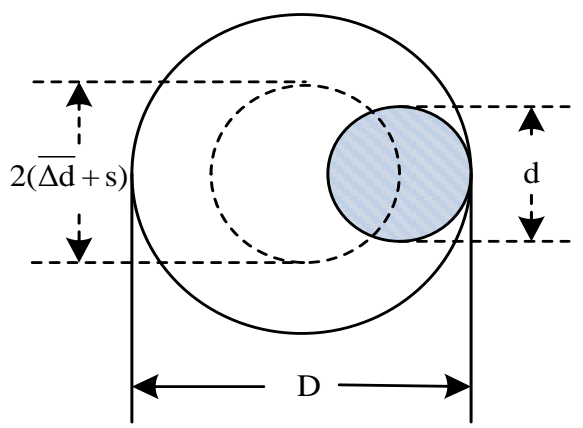

Fig. 5 Schematic of error

\subsection{Match and save jack indexes}

Bwlabel calculates every jack's center, but it has not built the relationship among different jacks, which belong to a same electronic component. Therefore, Bwlabel cannot be applied to production directly. A resolution is put forward that when producing a certain type of PCB, build the relationship artificially and save it, which is foundation of automatic positioning.

A PCB as Fig. 4 shows should be assembled into different electronic components, such as relay, fuse, fan capacitor, safety capacitor, buzzer, connector, and so on. All of them need 2 or more than 2 jacks. Fig. 6 shows the arrangement of jacks of 4 kinds of electronic components.

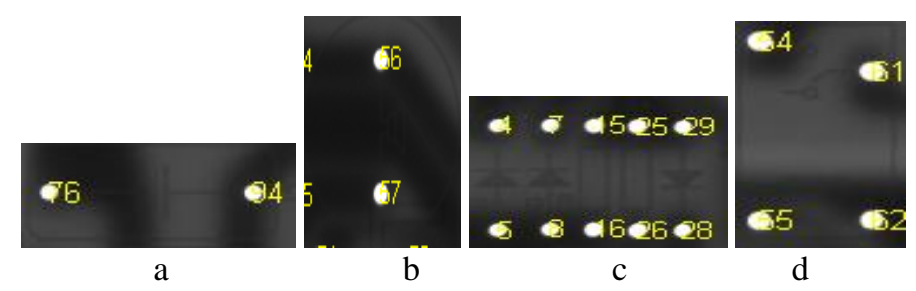

Fig. 6 Arrangement diagram of component jacks

Known by observation, all electronic components on the PCB are parallel to the surface. Therefore, if both the location and the direction of a piece of electronic component are determined, it can be positioned. For 2-pin-component positioning, only need to save central coordinates of the corresponding 2 jacks and the jack center connection angle. For those having more than 2 pins, select any two pins then do the same work as 2-pincomponent positioning.

According to the character of Bwlabel and repeatability of the result, a same jack on the same type of PCB may has a same index and different central coordinate in different images (make sure the image has no rotation). So the key work is to match the indexes which belong to a same piece of component, and link the central coordinates to the indexes. A program can do this automatically. 
A safety capacitor, whose corresponding jack indexes are 76, 94, should be assembled into a PCB shown as Fig. 6a. In Fig. 6b, a buzzer is needed, indexes are 56, 57. In Fig. 6c, a diode, indexes 4, 5. In Fig. 6d, a relay, indexes 54, 55, 61, 62. Select 2 indexes among them, for example 54, 55. Calculate the jack center connection angle of every 2 jacks and save the result to Access database (see Table. 3).

Table. 3 Positioning data

\begin{tabular}{cccc}
\hline Component & Jack 1 & Jack 2 & Angle [rad] \\
\hline $\begin{array}{c}\text { Safety } \\
\text { capacitor }\end{array}$ & 76 & 94 & 0 \\
Buzzer & 56 & 57 & $\pi / 2$ \\
Diode & 4 & 5 & $-\pi / 2$ \\
Relay & 54 & 55 & $\arctan \frac{Y_{55}-Y_{54}}{X_{55}-X_{54}}$ \\
$\ldots$ & $\ldots$ & $\ldots$ & $\ldots$ \\
\hline
\end{tabular}

\section{Simulation Results}

When produce a certain type of PCB at first time, initialize the data in Table. 1 and Table.3, and save to Access database. After that, trigger the camera and run the program by infrared signal. The program can call the data in database and calculate the positioning data automatically, which achieves the automatic positioning process. The whole process of automatic PCB jacks positioning is shown as Fig. 7 below.

An automatic PCB jack positioning system is developed based on Matlab R2009a, connecting the Access database to save and call the matched indexes and lens distortion data (see Fig. 8).

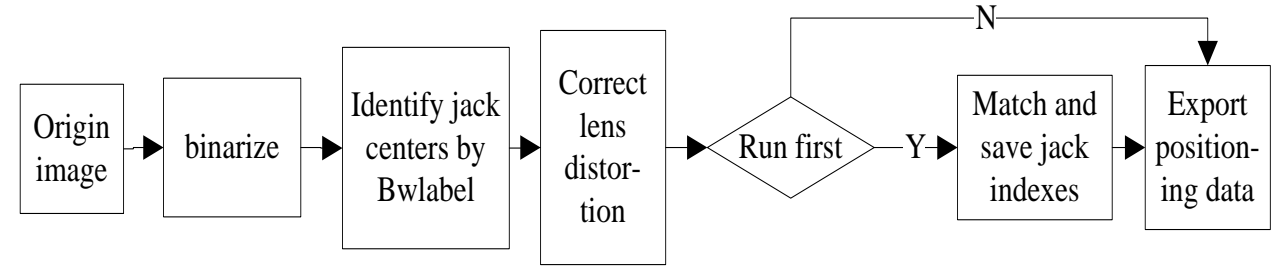

Fig. 7 Flow chart of automatic positioning 


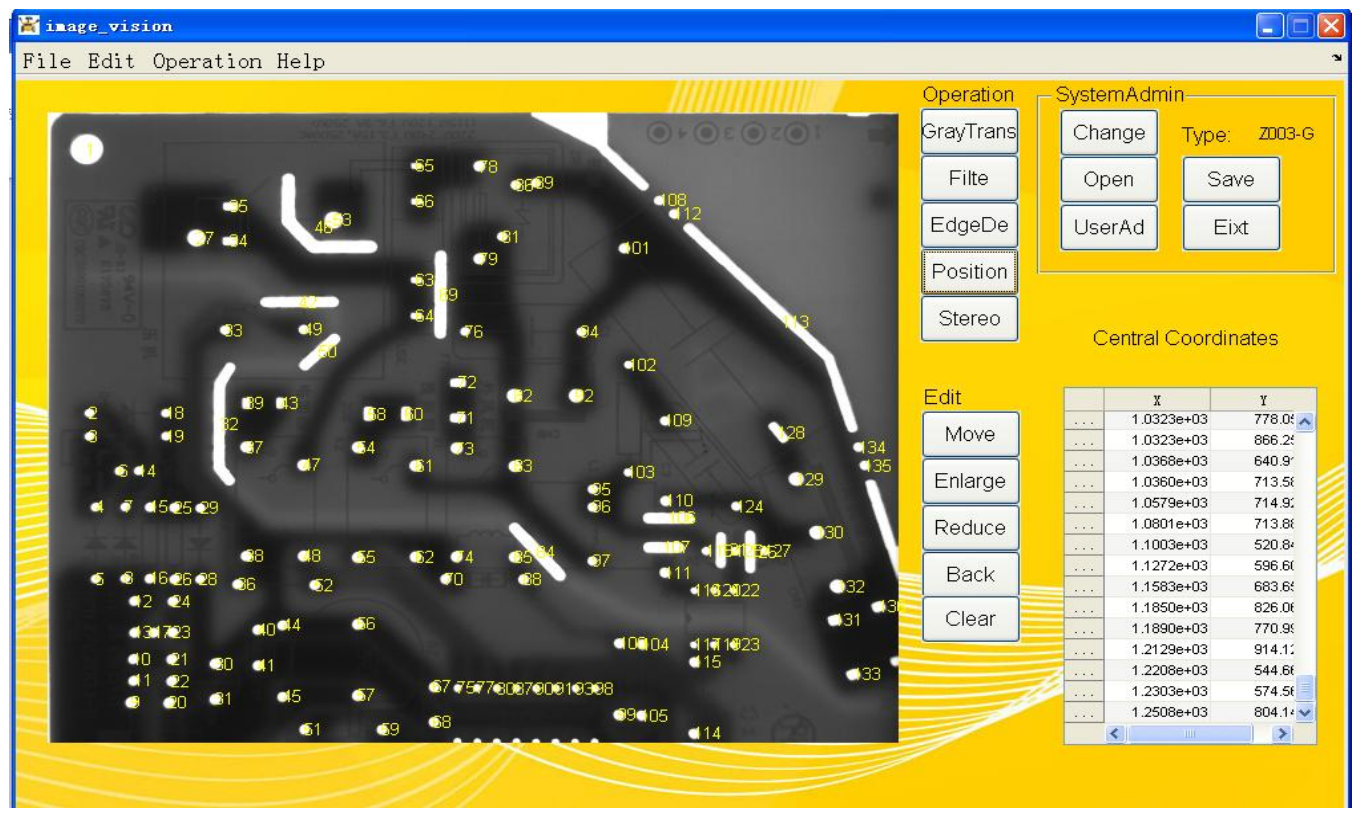

Fig. 8 Main system interface

When take the photo of PCB for the first time (take the GM127CZ003-G for example), the jack indexes should be matched and saved artificially. After that, for every other PCB which has the same type, the system will calculate the central coordinates of every jack, put them in a table and export the table (see Table. 4).

Table. 4 Automatic positioning result

\begin{tabular}{cccc}
\hline Component & Jack 1 & Jack 2 & $\begin{array}{c}\text { Angle } \\
{[\mathrm{rad}]}\end{array}$ \\
\hline Safety Capacitor & $(54.95,31.19)$ & $(70.44,31.32)$ & 0 \\
Buzzer & $(40.86,72.71)$ & $(40.96,83.06)$ & 1.56 \\
Diode & $(6.45,55.97)$ & $(6.43,66.39)$ & -1.57 \\
Relay & $(40.95,47.70)$ & $(41.08,63.59)$ & 1.56 \\
$\ldots$ & $\ldots$ & $\ldots$ & $\ldots$ \\
\hline
\end{tabular}

\section{Conclusion}

Machine vision technique is applied to PCB production. Identify the PCB jack centers by $B$ wlabel algorithm and make use of distortion model to correct the result. The accuracy can meet the production requirement when the jack diameter is greater than $2.32 \mathrm{~mm}$. For GM127CZ003-G type PCB, the average time of image processing is $0.21 \mathrm{~s}$, which is well real-time. A method, matching and saving the jack indexes and calculating the jack center connection angle, to describe the location and direction of electronic components is put forward, which achieves the automatic positioning process. Develop the automatic PCB jack center positioning system based on Matlab, which generates the complete positioning data. The data can be used in automatic assembly operation by manipulator. Besides, in the whole process, set the origin $(0,0)$ at the positioning jack at the upper left corner, which is not flexible enough. What's more, the position accuracy can be improved further, so that the minimum diameter can be smaller and more electronic components can be positioned automatically. 


\section{Acknowledgment}

This work is supported by the National Natural Science Foundation of China, NO: 31201135 , Combination of production and research project of Guangdong Provincial Ministry of Education, NO: 2011B090400160. The corresponding author of the paper is Jian-guo Chen (email: cjg63@qq.com).

\section{References}

[1] National Development and Reform Commission, 2008, Information on http://wenku.baidu.com/view/4d9f0dd8a58da0116c1749b7.html.

[2] He Wei-zhong, 2006, The Image Orientation System for Machining PCB, Chongqing: Chongqing University.

[3] Chen Ke-yong, 2007, Automatic Optical Positioning Technology in Industrial Automatization, Fujian: Xiamen University.

[4] Xia Qi, Zhou Ming-cai, WANG Hong-sheng, et al, 2004, Vision alignment system in automatic high precision chip mounter and its image processing, Optical Technique, 30(2): 146-149.

[5] Wang Hong, Wang Shi-gang, Xu Wei, et al, 2001, Angle Locating of Printed Circuit Board Based on Fractal Theory, Journal of Shanghai Jiaotong University, 35(12): 1781-1784.

[6] Jiang Tao, Wang An-lin, Wang Shi-gang, et al, 2005, An Error Correction Method for PCB Based on Machine Vision, Journal of Shanghai Jiaotong University, 39(6): 945949.

[7] Huang Yong-lin, 2010, Key Technologies and Realization of PCB Opto-electronic Hole Checker, Sichuang: University of Electronic Science and Technology.

[8] Zhu Yan, Duan Zhe-min, 2007, Application of Machine Vision Technique in the Identification for Welding Spots of Printed Circuit Board (PCB), Science Technology and Engineering, 7(6): 1045-1051.

[9] Xie Hong-fei, Zhang Xian-ming, Kuang Yong-cong, et al, 2011, Intelligent Detection of Solder Joints on Printed Circuit Boards, Optics and Precision Engineering, 19(9): 2154-2162.

[10] Su Lei, Zhang Guang-ming, Wei Xiao-dong, 2009, Design and Research of Machine Vision Based Defect Inspection System on Printed Circuit Board, Machine Tool \& Hydraulics, 37(8): 294-296.

[11] Jean-Yves Bouguet, 2010, Information on http://www.vision.caltech.edu/bouguetj/calib_doc.

[12]Fishgumuxing, 2010, Information on http://wenku.baidu.com/view/a1b7e17931b765ce050814a3.html.

[13] Wang Li, Zhang Mao-qing, Wang Hong-dong, 2009, Image Processing Algorithm Study of Surface-Mount Machine Element Positioning Based on Machine Vision, Electrical \& Electronics, (1): 57-59.

[14] Rafael C. Gonzalez, Richard E. Woods, Steven L. Eddins, 2009, Digital Image Processing Using MATLAB, Beijing: Publishing House of Electronics Industry: 289295.

[15] Li Ting, Liu Ning, 2012, Study on circle locating technology based on machine vision, Computer Engineering and Applications, 48 (9): 153-156.

[16] Gary Bradski, Adrian Kaehler, 2009, Learning OpenCV: Computer Vision with the OpenCV Library, CA: O'Reilly Media: 406-413.

[17] Liu Rui-zhen, Yu Shi-qi, 2007, OpenCV Tutorials-The Basics. Beijing: Beijing University of Aeronautics and Astronautics Press: 393-395. 